\title{
Loxoscelismo cutáneo y cutáneo-visceral: Revisión sistemática
}

\author{
Juan J. Manríquez M. y Sergio Silva V.
}

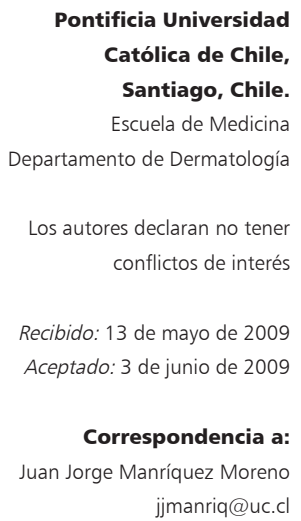

\section{Cutaneous and visceral loxoscelism: A systematic review}

Introduction: Loxoscelism represents a major public health problem for which there are no standard therapeutic interventions. Objective: To review available scientific evidence on management of Loxoscelism Method: Systematic review of clinical studies. The search included multiple databases (Medline, Lilacs, Embase, Web of Sciences, Cinahl, Pre-Cinahl, Paperfirst, Proceedingsfirst, Dissertations and Theses, Toxline, Cochrane Library), handsearch of references, and contact with experts. Results: Three clinical trials of poor methodological quality were identified from 5,207 references found. One trial $(n=31)$, concluded that the use of dapsone was associated with fewer local complications than surgical treatment. A second study $(n=46)$, concluded that the use of dapsone was superior to clorfenamine for skin lesions. A third study $(n=95)$ concluded that there was no differences between the use of oral dapsone, antivenom against anti-Loxosceles reclusa or a combination of both. Conclusions: There is insufficient evidence based on good quality studies to recommend treatment guidelines for individuals with skin or visceral loxoscelism.

Key words: Loxoscelism, systematic review, evidence-based medicine.

Palabras clave: Loxoscelismo, revisión sistemática, medicina basada en evidencia.

\section{Introducción}

$\mathrm{E}$ 1 loxoscelismo, cuadro clínico resultante de la mordedura de la araña del género Loxosceles, constituye un importante problema de salud pública en varios países sudamericanos, especialmente en regiones de Argentina, Perú, Chile y Brasil. El año 2005, el Centro de Información Toxicológica de la Universidad Católica de Chile, atendió 2.831 llamados telefónicos sobre pacientes con sospecha de loxoscelismo, siendo confirmados 287 de éstos ${ }^{1}$.

La distribución de las distintas especies de arañas Loxosceles es bastante amplia, describiéndose 13 especies en Norteamérica ${ }^{2,3}$, siendo Loxosceles reclusa la mejor conocida y responsable de la mayoría de envenenamientos en esta zona. En Centroamérica se describen 38 especies, mientras que en Sudamérica se describen 34 especies de arañas género Loxosceles, la mayoría de ellas endémicas en esta zona ${ }^{2}$.

Tres especies sudamericanas: Loxosceles laeta (ampliamente distribuida en esta parte del continente), Loxosceles intermedia (Brasil, Argentina) y Loxosceles gaucho (Brasil), son las especies más frecuentemente reportadas como causantes de loxoscelismo ${ }^{4,5}$, posiblemente por la mayor peligrosidad de su veneno (especialmente L. laeta), comparado con las especies norte y centroamericanas ${ }^{2}$.

En el resto del mundo, el género Loxosceles no está tan bien representado. En regiones Mediterráneas se encuen- tra Loxosceles rufescens, mientras que en África existen doce especies conocidas. En China se han reportado dos especies $^{2,3}$, y en zonas específicas de Australia se han encontrado poblaciones de L. rufescens ${ }^{6}$.

El veneno de esta araña es dermo-necrótico y víscerotóxico ${ }^{7,8}$. Es rico en enzimas de bajo peso molecular, específicamente esfingomielinasa, hidrolasa, hialuronidasa, lipasa, colagenasa, fosfatasa alcalina, proteasas, metaloproteasas, entre otras. El principal componente tóxico es la esfingomielinasa $\mathrm{D}$, que interactúa con las membranas celulares y otros elementos tisulares desencadenando alteraciones que involucran el sistema del complemento y activación de neutrófilos ${ }^{7,8}$.

La arañas del genero Loxosceles son las únicas capaces de generar necrosis cutánea, fundamentalmente por la presencia de la enzima esfingomielinasa $\mathrm{D}^{9-11}$, la que sólo se ha identificado en otro género de arañas Sicarius y varias bacterias ${ }^{2}$.

Asociado a la mordedura de esta araña, se han descrito dos cuadros clínicos:

- El primero y más frecuente $(83,3 \%$ del total según Schenone, 2003) $)^{12}$, denominado loxoscelismo cutáneo, es un cuadro limitado, generalmente benigno, caracterizado por dolor, edema y eritema dentro de las primeras 6 a 8 horas de la mordedura, que progresa a un halo de vasoconstricción e isquemia que se extiende alrededor de la lesión. Se puede formar una ampolla, y luego de varios días luego de la mordedura, 
la lesión toma un color azulado-violáceo (Figuras 1 y 2), de consistencia dura y con un centro hundido, a lo que sigue ulceración y necrosis del área afectada. La mordedura tiende a curar por segunda intención en 6 a 8 semanas $^{12,13}$. Se ha descrito también una variante únicamente edematosa de loxoscelismo cutáneo ${ }^{14}$ (Figura 3).

- El cuadro de loxoscelismo visceral se caracteriza por la presencia de compromiso sistémico, eventualmente letal, caracterizado por hemólisis, hematuria, hemoglobinuria, ictericia, fiebre e insuficiencia renal, que ocurre generalmente transcurridas las primeras 24 horas de la mordedura ${ }^{12}$.

Gran variedad de tratamientos han sido descritos para el manejo del cuadro de loxoscelismo cutáneo y visceral, entre ellos, corticosteroides, dapsona, colchicina, oxígeno hiperbárico, ex-sanguíneo-transfusión, antimicrobianos, electricidad, cirugía, medicamentos vasodilatadores, antihistamínicos, anticoagulantes, y suero anti-loxosceles. Sin embargo, no existe en la actualidad consenso sobre el mejor esquema de tratamiento, en sujetos afectados por este cuadro ${ }^{2,15}$.

La menor importancia relativa de este problema en países desarrollados se ha traducido en la existencia de una cantidad limitada de información publicada al respecto. A pesar de lo anterior, es importante destacar que en los últimos años se han publicado dos revisiones narrativas sobre el tema en revistas internacionales. La primera, realizada por Hogan y cols ${ }^{15}$ y la segunda, publicada por Swanson y cols en The New England Journal of Medici$n e^{16}$, lo que refleja un creciente interés en este problema.

Las revisiones mencionadas, si bien constituyen una fuente de información importante, adolecen de las limitaciones propias de las revisiones narrativas ${ }^{17-20}$. Además, por las características del problema, existe una probabilidad alta de que la información existente no se encuentre fácilmente accesible. Es posible que este tipo de información se publique en revistas no incluidas en las principales bases de datos, así como también puede existir información que no haya sido publicada ${ }^{21}$. La realización de revisiones no sistemáticas, aumenta la posibilidad de que la información presentada pueda contener sesgo proveniente de la opinión e intereses del autor, un problema común en este tipo de publicaciones ${ }^{22}$.

La araña Loxosceles se encuentra globalmente distribuida y se asocia a lesiones cutáneas necróticas, hemólisis, falla renal e incluso muerte. En Chile, Perú y Brasil constituye un problema relevante de salud pública, debido a la mayor tasa de compromiso cutáneo-visceral asociado a $L$. laeta, comparativamente mayor que el observado con $L$. reclusa, Loxosceles arizonica, L. rufescens o Loxosceles deserta, presentes en otros países del hemisferio norte y Europa. La ausencia de este problema en la mayoría de los

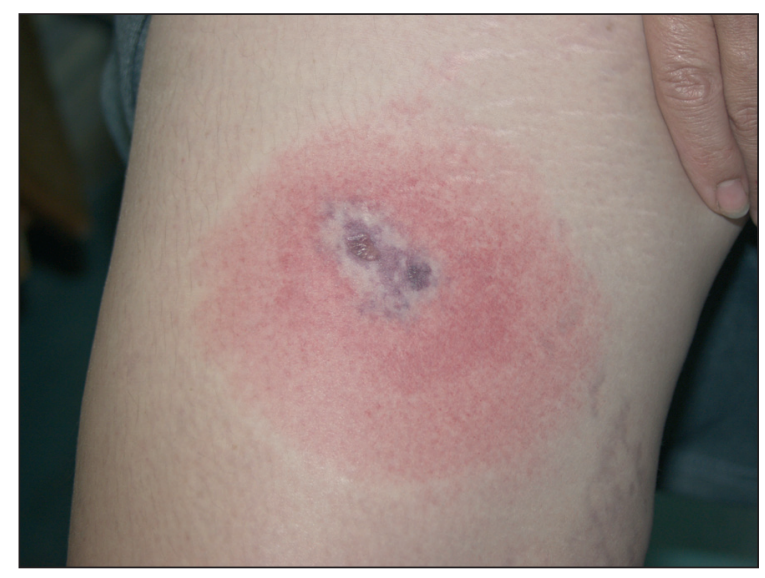

Figura 1. Placa livedoide en desarrollo.

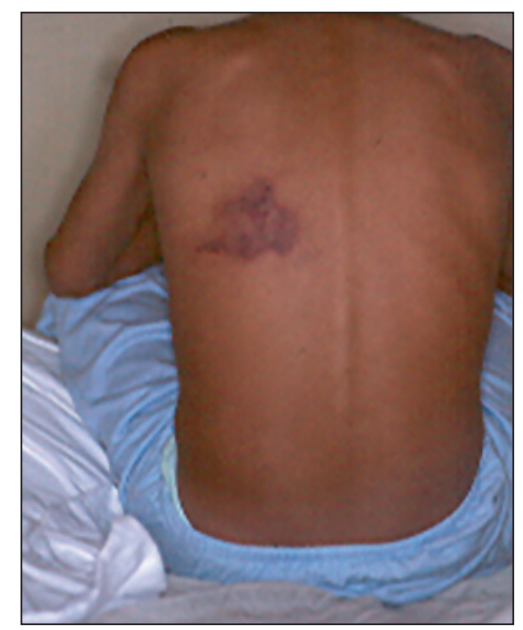

Figura 2. Placa livedoide en desarrollo.

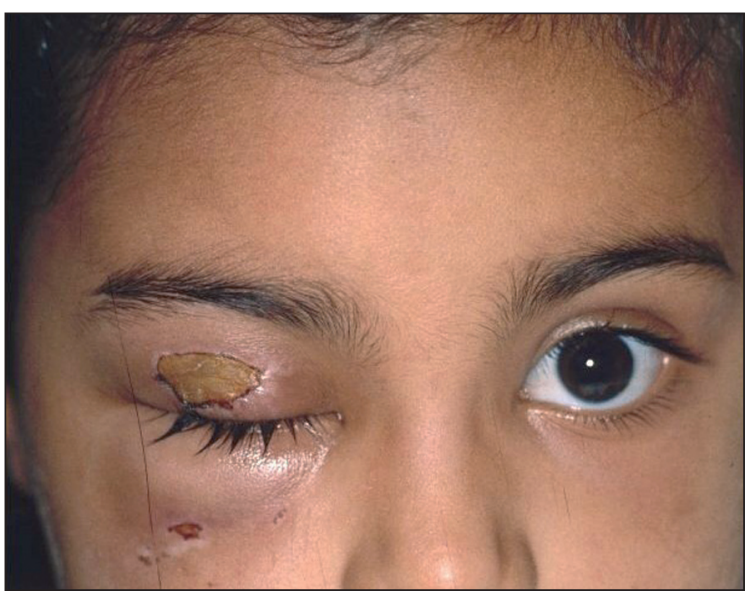

Figura 3. Loxoscelismo cutáneo en su forma edematosa. 
países en que se desarrolla gran parte de la investigación clínica, hace que el número de estudios sobre la efectividad de las medidas terapéuticas para este accidente sea escaso. Es probable que de existir información, no haya sido suficientemente difundida, por el menor interés de las revistas de mayor impacto. Incluso existe la posibilidad que parte de la investigación no haya llegado a publicarse, o bien sea en revistas en lenguaje distinto al inglés (español o portugués) de bajo impacto, y generalmente no indizadas en las bases de datos biomédicas tradicionales, como Medline o Embase.

El objetivo de este estudio fue buscar sistemáticamente la evidencia científica, proveniente de la investigación clínica en humanos, relacionada al manejo de la mordedura de araña género Loxosceles, jerarquizando la información proveniente de estos estudios, de acuerdo a diseño metodológico y calidad del método, de modo de establecer el nivel de evidencia de cada intervención.

\section{Material y Método}

El problema a abordar fue estructurado en forma de pregunta clínica, mediante la definición explícita del tipo de paciente o población, la intervención a evaluar, las posibles comparaciones y los "outcomes" u observables de interés.

Tabla 1. Intervenciones incluidas en la revisión
Estrategias terapéuticas
1. Oxígeno hiperbárico
2. Uso de hielo
3. Suero anti-loxosceles
4. Dapsona
5. Antihistamínicos
6. Antimicrobianos
7. Dextrán
8. Corticosteroides
9. Heparina
10. Nitroglicerina
11. Cirugía
12. Ácido acetilsalicílico
13anguineo-transfusión

Población de interés. En este estudio la población de interés estuvo compuesta por todos los pacientes afectados por mordedura de loxosceles de cualquier especie. Fueron incluidos sólo estudios clínicos, realizados en seres humanos.

Intervenciones. Las intervenciones estudiadas se resumen en la Tabla 1. Previo a la ejecución de la revisión, asumimos que no habían intervenciones de efecto demostrado mediante estudios de alta calidad metodológica (y que por tanto pudiesen considerarse como estándar), por lo que incluimos como comparadores válidos idealmente el placebo, o en su defecto un grupo control sin intervención. Anticipando la posibilidad de encontrar una cantidad insuficiente de estudios controlados, se incluyeron en la búsqueda todos los diseños epidemiológicos posibles (series de casos, estudios de caso-control, estudios de cohorte, ensayos clínicos).

Definición de outcome. Los outcomes u observables de interés fueron ordenados de acuerdo a una escala de medidas de outcome, adaptada y modificada de la utilizada por el National Health and Medical Research Council (NHMRC) Australia ${ }^{23}$. Aquellos outcomes considerados clínicamente relevantes (outcomes primarios) fueron clasificados como nivel 1; por otro lado, aquellos outcomes subrogados (outcomes secundarios: outcome intermedios posiblemente predictores de outcomes clínicamente relevantes) fueron clasificados como nivel 2 (Tabla 2).

\section{Búsqueda de la información}

Bases de datos. Las bases de datos analizadas, y las estrategias utilizadas para búsqueda de estudios se describen más abajo. No hubo restricción según idioma de publicación de los artículos originales. Las búsquedas fueron realizadas hasta noviembre de 2007.

Tabla 2. Escala de medidas de outcome utilizadas en la revisión (Adaptada y modificada de ${ }_{23}$ National Health and Medical Research Council)

Outcomes Primarios: Nivel 1
- Tasa de mortalidad
- Tasa de insuficiencia renal
- Necesidad de cuidados intensivos
Outcomes Secundarios: Nivel 2
- Estadía hospitalaria
- Tasa de formación de úlcera
- Tasa de necesidad de injerto
- Tasa de cicatrización anómala
- Dolor local
- Grado de edema




\section{Medline}

1. arachnidism [ALL]

2. spider AND venom* [ALL]

3. loxoscelism* [ALL]

4. loxocelism* [ALL]

5.1 or 2 or 3 or 4

\section{Lilacs}

1. Tw arachnidism

2. Tw spider AND venom\$

3. Tw loxoscelism\$

4. Tw loxocelism\$

5.1 or 2 or 3 or 4

\section{Embase}

1. loxoscel\$.mp. [mp=title, abstract, subject headings, heading word, drug trade name, original title, device manufacturer, drug manufacturer name]

2. loxocel\$.mp. [mp=title, abstract, subject headings, heading word, drug trade name, original title, device manufacturer, drug manufacturer name]

3. spider venoms.mp. [mp=title, abstract, subject headings, heading word, drug trade name, original title, device manufacturer, drug manufacturer name]

4. arachnidism.mp.

5. 1 or 2 or 3 or 4

Web of Sciences

1. $\mathrm{TS}=$ loxocelism*

DocType $=$ All document types; Language $=$ All languages; Databases=SCI-EXPANDED, SSCI, A\&HCI; Timespan $=1988-2007$ 11

2. TS=loxoscelism*

DocType $=$ All document types; Language $=$ All languages; Databases=SCI-EXPANDED, SSCI, A\&HCI; Timespan $=1988-2007$

3. $\mathrm{TS}=($ spider AND venom $*)$

DocType $=$ All document types; Language $=$ All languages; Databases=SCI-EXPANDED,

SSCI, A\&HCI; Timespan=1988-2007

4. $\mathrm{TS}=$ arachnidism

DocType $=$ All document types; Language $=$ All languages; Databases=SCI-EXPANDED, SSCI, A\&HCI; Timespan $=1988-2007$

5. \#4 OR \#3 OR \#2 OR \#1

\section{Cinahl}

1. $\mathrm{TX}=$ arachnidism

2. $\mathrm{TX}=($ spider AND venom $*)$

3. $\mathrm{TX}=$ loxoscelism*

4. $\mathrm{TX}=$ loxocelism $*$

5. \#4 OR \#3 OR \#2 OR \#1
Pre-Cinahl

1. $\mathrm{TX}=$ arachnidism

2. $\mathrm{TX}=($ spider $\mathrm{AND}$ venom* $)$

3. $\mathrm{TX}=$ loxoscelism*

4. $\mathrm{TX}=$ loxocelism*

5. \#4 OR \#3 OR \#2 OR \#1

\section{Paperfirst}

1. kw: arachnidism

2. kw: spider w venom*

3. kw: loxoscelism*

4. kw: loxocelism*

5. \#4 OR \#3 OR \#2 OR \#1

Proceedingsfirst

1. kw: arachnidism

2. kw: spider w venom*

3. kw: loxoscelism*

4. kw: loxocelism*

5. \#4 OR \#3 OR \#2 OR \#1

Dissertations and theses

1. arachnidism [ALL]

2. spider AND venom* [ALL]

3. loxoscelism* [ALL]

4. loxocelism* [ALL]

5. 1 or 2 or 3 or 4

Toxline

1. arachnidism [ALL]

2. spider AND venom* [ALL]

3. loxoscelism* [ALL]

4. loxocelism* [ALL]

5. 1 or 2 or 3 or 4

\section{Cochrane Library}

1. arachnidism [ALL]

2. spider AND venom* [ALL]

3. loxoscelism* [ALL]

4. loxocelism* [ALL]

5. 1 or 2 or 3 or 4

\section{Referencias de estudios publicados}

Se realizó además búsqueda manual de referencias de estudios encontrados, y de una serie de artículos de revisión seleccionados.

\section{Literatura no publicada}

Se contactó con expertos en el área.

\section{Selección de estudios}

Uno de los investigadores (JM) realizó las búsquedas $\mathrm{y}$ ambos autores, en forma ciega e independiente, seleccionaron artículos que fueron incluidos en este estudio. La 
extracción de datos y análisis de calidad fue realizado por uno de los autores (JM), según formulario pre-establecido. Cualquier discrepancia fue resuelta mediante discusión entre los revisores, o en caso de necesidad, una tercera parte.

\section{Niveles de evidencia y evaluación de calidad de los estudios}

- Los estudios seleccionados fueron clasificados según nivel de evidencia, en una escala de I a IV, adaptada de la usada por el NHMRC ${ }^{23}$, en base a la probabilidad de reducir el riesgo de sesgo, según el diseño metodológico elegido. De este modo la evidencia proveniente de una revisión sistemática de ensayos clínicos fue catalogada como nivel I; aquella proveniente de un ensayo clínico randomizado como nivel II; aquella proveniente de un ensayo clínico seudo-randomizado como nivel III-1; aquella derivada de estudios casocontrol o cohorte o una revisión sistemática de este tipo de estudios como tipo III-2; finalmente la información proveniente de series de casos fue clasificada como nivel IV.

- La calidad de cada estudio fue evaluada según un listado de criterios, adaptados y modificados por aquellos utilizados por la NHMRC ${ }^{23}$, y la Colaboración Cochrane ${ }^{24}$. Esta evaluación se hizo basado en la evaluación del diseño metodológico del estudio, según lo reportado en cada artículo o, cuando fue posible, según la información adicional aportada por el autor mediante contacto personal. La lista de criterios evaluados, según el diseño de cada estudio, se presenta en la Tabla 3. Cada estudio se clasificó como grado A si el criterio se cumplió correctamente, o como grados B, C, o D según el criterio no estuviese claro, fuese inadecuado, o no hubiese sido cumplido, respectivamente. Los estudios correspondientes a series de casos no fueron evaluados en cuanto a calidad, por la inexistencia de pautas validadas para este fin. Al momento de la ejecución de esta revisión la Declaración STROBE no había sido publicada ${ }^{25}$.

Se jerarquizó la calidad de los estudios evaluados como sigue: como grado A (bajo riesgo de sesgo) si todos los criterios evaluados recibieron una $\mathrm{A}$. Como grado $\mathrm{B}, \mathrm{C}$ o $\mathrm{D}$ se clasificó aquellos estudios con uno o más criterios clasificados como B, C, o D, respectivamente.

\section{Análisis y resumen de la evidencia encontrada}

Se planificó resumir la evidencia encontrada, en caso que los estudios encontrados los permitiesen (estudios controlados y homogéneos -según test $\chi^{2}$ heterogeneidad-, en forma de meta-análisis según un modelo de efectos aleatorios, utilizando OR (odds ratio) como medida de magnitud, e intervalo de confianza al 95\%, como medida de precisión de los resultados obtenidos.

Ante la ausencia de las condiciones pertinentes entre los estudios encontrados, la síntesis de la información se presenta en forma narrativa, en forma de revisión sistemática cualitativa, sin meta-análisis.

\section{Resultados}

\section{Descripción de los estudios}

De un total de 5.207 referencias obtenidas a través de las búsquedas en las diferentes fuentes de información citadas, un total de 66 fueron seleccionadas para análisis a texto completo ${ }^{26-91}$. Sólo 10 estudios cumplieron los criterios de inclusión y fueron incluidos en esta revisión ${ }^{82-91}$.

El resultado de la búsqueda en las distintas fuentes de información consultadas, así como la distribución de artículos inicialmente seleccionados para análisis a texto completo, y estudios finalmente incluidos por fuente, se resume en la Tabla 4. Las razones de exclusión de los artículos inicialmente seleccionados para análisis a texto completo fueron: artículos correspondientes a reporte aislado de $\operatorname{casos}^{30-33,35,41,44,47,49,51,56,57,62,63,74,75,80,81}$, estudio sobre prevención ${ }^{66}$, revisiones sistemáticas sin información pertinente ${ }^{54,64,77}$, revisión narrativa ${ }^{58}$, estudios en modelo animal ${ }^{36,37}$, carta al editor expresando opiniones personales ${ }^{38}$, estudios descriptivos y series de casos sin análisis de efectividad de tratamien$\operatorname{tos}^{26-29,34,39,40,42,43,45,46,48,50,52,53,55,59-61,65,67-73,76,78,79}$.

De los 10 estudios incluidos en esta revisión, siete correspondieron a series de casos, que evaluaron la efectividad de dapsona ${ }^{82,83}$, clorfenamina ${ }^{84}$, aplicación directa de corriente eléctrica de alto voltaje ${ }^{85}$, oxigeno hiperbári$\mathrm{co}^{86}$, tratamiento quirúrgico, y la aplicación de múltiples tratamientos ${ }^{88}$. En la Tabla 5 se observa un resumen de las características principales de estos estudios.

Sólo tres de los 10 estudios fueron ensayos clínicos, dos de ellos randomizados, que compararon la efectividad de dapsona combinado con suero anti-L. reclusa versus cada tratamiento en forma individual ${ }^{89}$, y la efectividad de dapsona versus clorfenamina ${ }^{90}$. El último estudio correspondió a un ensayo clínico no randomizado, que comparó la efectividad de dapsona versus tratamiento quirúrgico precoz ${ }^{91}$. En la Tabla 6 se observa un resumen de las características principales de estos estudios.

\section{Calidad metodológica}

En general, la calidad metodológica de todos los estudios incluidos fue pobre. De los 10 estudios, sólo tres correspondieron a ensayos clínicos, dos de ellos randomizados (nivel de evidencia II) y uno de tipo norandomizado (nivel de evidencia III-1). No se encontró estudios de cohorte, ni estudios caso-control. Los restantes 
siete estudios (series de casos-nivel de evidencia IV) no fueron analizados en su calidad metodológica, por la falta de pautas validadas para este efecto.

En cuanto a los ensayos clínicos incluidos, el análisis de calidad fue como sigue:

- Randomización y sesgo de selección: El proceso de randomización en general y el ocultamiento de la secuencia de randomización en particular, es la medida más importante para garantizar la minimización de sesgos en la ejecución de un ensayo clínico ${ }^{92}$. En ninguno de los dos ensayos clínicos randomizados, ni el método de generación de la secuencia de randomización, ni el método empleado para ocultar dicha secuencia fue descrito $^{89,90}$. El tercer ensayo clínico no fue descrito como randomizado ${ }^{91}$.

- Ciego y sesgo de detección: En ninguno de los tres ensayos, ni los sujetos participantes, los clínicos tratantes, adjudicadores de eventos, ni analistas de datos fueron descritos como ciegos.

- Manejo de las pérdidas y abandonos: Ninguno de los tres ensayos incluyó un correcto reporte de las pérdidas y abandonos, ni de las causas de éstos. Asimismo, ninguno de los tres ensayos reportó análisis de los datos según principio de intención de tratar.

\section{Tipos de loxoscelismo, tiempo de seguimiento y outcomes evaluados}

Los 10 estudios incluidos fueron realizados entre sujetos con loxoscelismo cutáneo, con diagnóstico clínico, variando los niveles de certeza diagnóstica, según la observación o no de la araña, la historia de una picadura o las características clínicas de la lesiones.

Sólo un estudio incluyó únicamente sujetos con diagnóstico confirmado de loxoscelismo, definido como la identificación de la araña causante por un entomólogo o investigador experto ${ }^{89}$. Un estudio incluyó sólo sujetos con lesiones cutáneas luego de uno a tres días de observación como método de confirmación diagnóstica ${ }^{86}$. Cuatro estudios consideraron como criterio de inclusión el diagnóstico de certeza (mediante la visualización de la araña) y el diagnóstico probable (combinación de características de la historia clínica y hallazgos al examen físico $)^{82,83,85,88}$. Los restante cuatro estudios ${ }^{84,87,90,91}$ incluyeron sujetos con diagnóstico clínico de loxoscelismo cutáneo, sin descripción del fundamento de este diagnóstico. La mayoría de los estudios siguieron los sujetos hasta la mejoría de las lesiones cutáneas, sin un período de seguimiento predeterminado al inicio del estudio.

Ningún estudio determinó efectividad de tratamientos en sujetos con loxoscelismo con compromiso visceral.

Ningún estudio evaluó outcomes primarios (de nivel 1). La mayoría de los estudios evaluaron la efectividad de distintas intervenciones, en los efectos locales de la mordedura, tales como tiempo para curación de herida,

\begin{tabular}{|ll} 
Tabla 3. Listado de criterios de calidad evaluados según diseño \\
metodológico
\end{tabular}

Tabla 4. Flujo de referencias analizadas, según fuente de obtención

\begin{tabular}{|lccc|}
\hline Base de datos & $\begin{array}{c}\text { Referencias } \\
\text { analizadas }\end{array}$ & $\begin{array}{c}\text { Artículos } \\
\text { analizados a } \\
\text { texto completo }\end{array}$ & $\begin{array}{c}\text { Artículos } \\
\text { finalmente } \\
\text { incluidos }\end{array}$ \\
\hline Medline & 3.039 & 45 & 5 \\
Lilacs & 200 & 32 & 7 \\
\hline Embase & 1.017 & 21 & 2 \\
\hline Web of Sciences & 360 & 14 & 4 \\
\hline Cinahl & 44 & 2 & 0 \\
\hline Pre-Cinahl & 0 & 0 & 0 \\
\hline Paperfirst & 0 & 0 & 0 \\
\hline Proceedingsfirst & 0 & 0 & 0 \\
\hline Dissertations and theses & 61 & 0 & 5 \\
\hline Toxline & 453 & 8 & 0 \\
\hline Cochrane Library & 11 & 0 & 0 \\
\hline Bases de ensayos clínicos en curso & 7 & 0 & 2 \\
\hline Búsqueda manual de referencias & 10 & 2 & 1 \\
\hline Contacto con expertos & 5 & 1 & 0 \\
\hline
\end{tabular}




\section{Tabla 5. Resumen de las características principales de las series de casos incluidas en la revisión}

\section{Estudio/Diseño Nivel de evidencia/calidad}

Escalante, $1999^{82}$

Serie de casos

(Nivel IV; calidad grado D)

$\begin{array}{ll}\text { Maguiña, 198783 } & \text { Sujetos con loxoscelis- } \\ \text { Serie de casos } & \text { mo cutáneo }(n=23 ; \\ \text { (Nivel IV; calidad grado D) } & \text { analizados 16) }\end{array}$

Población

Niños con loxoscelismo

cutáneo $(n=11)$

analizados 16)
Intervención (S)

Dapsona $1 \mathrm{mg} / \mathrm{kg}$, con

o sin paracetamol o

cloxacilina

Dapsona 100 mg/día más otras co-intervenciones múltiples

\section{Outcome (S) (Nivel)}

\section{Efectividad}

Evolución de lesiones

cutáneas

(outcome nivel 2)
Evolución de lesiones cutáneas (outcome nivel 2)

\section{0 sujetos con mejoría Ninguno completa}

De 10 sujetos con placa livedoide, 1 resultó sin secuelas y 8 con cicatriz.

De 5 sujetos con lesiocionaron sin secuelas y 3 con costra residual

Schenone, $1959^{84}$

Serie de casos

Sujetos con loxoscelismo cutáneo $(\mathrm{n}=25)$ nes violáceas, 2 evolu-
Clorfenamina intramuscular o subcutánea, según protocolo
Evolución del dolor y

edema

(outcome nivel 2)
Todos experimentaron disminución del dolor en 2 a 10 días. 18 sujetos tuvieron "reducción importante del edema" en 24 horas
Osborn, $1991^{85}$

Serie de casos

(Nivel IV; calidad grado D)

\section{Sujetos con loxoscelis-} mo cutáneo ( $n=147)$
Aplicación directa de corriente eléctrica de alto voltaje, con o sin corticosteroides, antimicrobianos y toxoide tetánico

\section{Oxígeno hiperbárico 2} a 2,5 atmósferas, por 7 días

(Nivel IV; calidad grado D)

Auer, $1974^{87}$

Serie de casos

(Nivel IV; calidad grado D)

\section{Sujetos con loxoscelis-} mo cutáneo $(n=14)$

Sujetos con loxoscelismo cutáneo $(n=33)$
Tratamiento quirúrgico precoz, tratamiento quirúrgico tardío o tratamiento médico

$\begin{array}{lll}\begin{array}{l}\text { Progresión de signos } \\ \text { locales y necesidad de } \\ \text { cirugía } \\ \text { (outcome nivel 2) }\end{array} & \begin{array}{l}\text { Ningún sujeto progresó } \\ \text { a necrosis ni formación } \\ \text { local de ampollas. Nin- } \\ \text { guno requirió cirugía }\end{array} & \text { Ninguno } \\ \begin{array}{l}\text { Evolución de lesión cu- } \\ \text { tánea y necesidad de } \\ \text { injerto }\end{array} & \begin{array}{l}\text { Todos con resolución } \\ \text { completa. Ningún suje- } \\ \text { to requirió injerto }\end{array} & \text { Ninguno } \\ \begin{array}{l}\text { No descrito } \\ \text { (outcome nivel 2) }\end{array} & \text { No descrito } & \text { No reportado } \\ \begin{array}{l}\text { Tiempo hasta mejoría. } \\ \begin{array}{l}\text { Tasa de cicatrización } \\ \text { (outcome nivel 2) }\end{array}\end{array} & \text { Sin diferencias } & \begin{array}{l}\text { Corticosteroides y dap- } \\ \text { sona asociados a retraso } \\ \text { en curación de herida. }\end{array}\end{array}$

Mold, $2004^{88}$

Serie de casos

(Nivel IV; calidad grado D)

\begin{tabular}{|c|c|c|c|}
\hline $\begin{array}{l}\text { Sujetos con loxoscelis- } \\
\text { mo cutáneo }(n=262)\end{array}$ & Múltiples & $\begin{array}{l}\text { Tiempo hasta mejoría. } \\
\text { Tasa de cicatrización } \\
\text { (outcome nivel 2) }\end{array}$ & Sin diferencias \\
\hline
\end{tabular}

Un sujetos experimentó dermatitis secundaria a dapsona

\section{No reportado} en curación de herida. dolor local, grado de edema, necesidad de cirugía, necesidad de injerto y cicatrización anómala (outcomes secundarios-nivel 2).

\section{Intervenciones}

\section{Dapsona}

Efectividad. Un ensayo clínico randomizado (Nivel II; calidad grado D; outcome nivel 2$)^{89}$, realizado entre sujetos con loxoscelismo cutáneo, atendidos antes de 96 horas de evolución desde la mordedura (media 33 horas), con diagnóstico confirmado por visualización directa de la araña por un entomólogo o investigador experto (sujetos confirmados y analizados 17; sujetos excluidos $78)$, demostró que no habría diferencias entre el uso de dapsona en dosis de $100 \mathrm{mg} /$ día, la combinación de suero anti-L. reclusa obtenido de conejos en dosis de 0,2 $\mathrm{ml}$ intra-lesional y dapsona $100 \mathrm{mg}$ diarios, o sólo suero anti-L. reclusa $0,2 \mathrm{ml}$ intra-lesional, en cuanto acortar el tiempo hasta la curación de la herida cutánea (todos los sujetos, salvo dos que fueron excluidos del análisis por presentar gran necrosis al inicio, lograron curación en $20 \pm$ 11 días; $p>0,05)$. Todos los sujetos recibieron además cotratamiento con eritromicina $400 \mathrm{mg}$ cuatro veces al día.

Un segundo ensayo clínico randomizado (Nivel II; calidad grado D; outcome nivel 2) ${ }^{90}$, realizado entre sujetos con loxoscelismo cutáneo $(n=46$; analizados $n=39$ ), atendidos entre 24 horas y 5 días después de la mordedura, demostró que el uso de dapsona $100 \mathrm{mg}$ /día durante 5 días, 


\begin{tabular}{|c|c|c|c|c|c|}
\hline $\begin{array}{l}\text { Estudio/diseño } \\
\text { Nivel de evidencia/calidad }\end{array}$ & Población & Intervención(s) & Outcome(s) (nivel) & Efectividad & Efectos adversos \\
\hline $\begin{array}{l}\text { Rees, } 1987^{89} \\
\text { Ensayo clínico randomizado } \\
\text { (Nivel II; calidad grado D) }\end{array}$ & $\begin{array}{l}\text { Sujetos con loxoscelis- } \\
\text { mo cutáneo ( } n=95 \\
\text { analizados } 17)\end{array}$ & $\begin{array}{l}\text { Dapsona } 100 \mathrm{mg} / \text { día vs } \\
\text { suero anti-Loxosceles } \\
\text { intralesional vs ambos } \\
\text { juntos; todos además } \\
\text { recibieron eritromicina } \\
\text { oral }\end{array}$ & $\begin{array}{l}\text { Tiempo hasta la mejoría } \\
\text { de la lesión cutánea } \\
\text { (outcome nivel 2) }\end{array}$ & $\begin{array}{l}\text { Excluyendo } 2 \text { sujetos } \\
\text { que presentaron necro- } \\
\text { sis, todos presentaron } \\
\text { curación en } 20 \pm 11 \\
\text { días }(p>0,05)\end{array}$ & No reportado \\
\hline $\begin{array}{l}\text { Maguiña, } 1997^{90} \\
\text { Ensayo clínico randomizado } \\
\text { (Nivel II; calidad grado D) }\end{array}$ & $\begin{array}{l}\text { Sujetos con loxoscelis- } \\
\text { mo cutáneo ( } n=46 \text {; } \\
\text { analizados } 39)\end{array}$ & $\begin{array}{l}\text { Dapsona } 100 \text { mg/día vs } \\
\text { clorfenamina } 4 \text { a } 8 \text { mg } \\
\text { dos veces al día }\end{array}$ & $\begin{array}{l}\text { Evolución de lesiones } \\
\text { cutáneas } \\
\text { (outcome nivel 2) }\end{array}$ & $\begin{array}{l}18 \text { de } 20 \text { sujetos tra- } \\
\text { tados con dapsona vs. } \\
10 \text { de } 19 \text { tratados con } \\
\text { clorfenamina curaron } \\
\text { sin úlcera ni necrosis }\end{array}$ & No reportado \\
\hline $\begin{array}{l}\text { Rees, } 1985^{91} \\
\text { Ensayo clínico no randomizado } \\
\text { (Nivel III-1; calidad grado D) }\end{array}$ & $\begin{array}{l}\text { Sujetos con loxoscelis- } \\
\text { mo cutáneo }(n=31)\end{array}$ & $\begin{array}{l}\text { Dapsona (dosis no des- } \\
\text { crita) vs tratamiento } \\
\text { quirúrgico }\end{array}$ & $\begin{array}{l}\text { Retraso en cicatrización } \\
\text { y cicatrización anómala } \\
\text { (outcome nivel 2) }\end{array}$ & $\begin{array}{l}5 \text { sujetos tratados con } \\
\text { cirugía experimentaron } \\
\text { retraso en cicatriza- } \\
\text { ción, y } 7 \text { cicatrización } \\
\text { anómala. Sólo } 1 \text { sujeto } \\
\text { presentó estos efectos } \\
\text { entre los tratados con } \\
\text { dapsona }\end{array}$ & $\begin{array}{l}1 \text { sujeto tratado con } \\
\text { dapsona experimentó } \\
\text { molestias digestivas }\end{array}$ \\
\hline
\end{tabular}

fue superior al uso de clorfenamina vía oral, en dosis de 4 a 8 mg cada 12 horas, en lograr evolución favorable de la herida cutánea (ausencia de úlcera o necrosis una vez finalizado el tratamiento), en 18 de 20 sujetos tratados con dapsona con evolución favorable, comparado con $10 \mathrm{de}$ 19 tratados con clorfenamina $(\mathrm{p}<0,02)$.

Un tercer ensayo clínico (Nivel III-1; calidad grado D; outcome nivel 2) ${ }^{91}$, realizado entre 31 sujetos con diagnóstico clínico de loxoscelismo cutáneo (mediante visualización de la araña o cuadro clínico compatible), demostró que el uso de dapsona (dosis y esquema de administración no descrito), seguido de tratamiento quirúrgico según evolución clínica, sería más efectivo que el tratamiento quirúrgico precoz (cierre primario o por segunda intención, con o sin injerto), en evitar el retraso de la cicatrización o cicatrización anómala, con 5 de 14 y 7 de 14 sujetos tratados con cirugía precoz con retardo la cicatrización y cicatrización anómala, comparado con 1 de 17 y 1 de 17, tratados con dapsona en forma inicial, respectivamente $(\mathrm{p}<0,05)$. Sólo un sujeto tratado con dapsona requirió cirugía posterior.

Tres series de casos, la primera ${ }^{82}$ (Nivel IV; calidad grado $\mathrm{D}$; outcome nivel 2$)$ realizada en niños $(\mathrm{n}=11$; edad rango 10 a 180 meses) con diagnóstico de loxoscelismo cutáneo (mediante observación de araña o cuadro clínico compatible) demostró que el tratamiento con dapsona 1 $\mathrm{mg} / \mathrm{kg}$ peso/día, durante 7 a 14 días según evolución, asociado a paracetamol en caso de dolor, o cloxacilina si hubiere signos de infección, sería efectivo en lograr mejoría completa de las lesiones cutáneas (10 sujetos presentaron resolución completa). La segunda serie ${ }^{83}$ (Nivel IV; calidad grado D; outcome nivel 2), realizada entre 23 sujetos (16 analizados) con loxoscelismo cutáneo (definido mediante observación de araña o cuadro clínico compatible), demostró que el uso de dapsona $100 \mathrm{mg}$ /día vía oral, administrada entre 5 y 20 días, sería efectiva en mejorar la evolución de la lesión cutánea ( 1 de 10 sujetos con placa livedoide presentó resolución completa, 8 de 16 formaron cicatriz sin úlcera y 1 de 10 evolucionó con úlcera; 2 de 5 sujetos con lesión violácea presentaron resolución completa y 3 de 5 evolucionaron con costra sin formación de úlcera; uno de 1 sujeto con cuadro edematoso presentó resolución completa).

Finalmente, una tercera serie ${ }^{88}$ (Nivel IV; calidad grado D; outcome nivel 2), realizada entre 262 sujetos con diagnóstico clínico de loxoscelismo cutáneo, tratados con múltiples intervenciones, demostró mediante análisis multivariado, que el uso de dapsona y corticosteroides sistémicos se asociarían a un retraso en la curación de la herida cutánea, y que dapsona se asociaría a un mayor riesgo de cicatrización.

Efectos adversos. Ninguno de los estudios reportó efectos adversos serios asociados al uso de dapsona. En uno de los ensayos 91 un sujeto presentó molestias gástricas. En otro estudio ${ }^{83}$ un sujeto presentó erupción cutánea.

\section{Clorfenamina}

Efectividad. Un ensayo clínico randomizado (Nivel 
II; calidad grado D; outcome nivel 2) ${ }^{90}$, demostró que el uso de clorfenamina vía oral, en dosis de 4 a $8 \mathrm{mg}$ cada 12 horas fue inferior a dapsona $100 \mathrm{mg}$ /día durante 5 días, en lograr evolución favorable de la herida cutánea (ver más arriba).

Una serie de $\operatorname{casos}^{84}$ (Nivel IV; calidad grado D; outcome nivel 2), realizada entre 25 sujetos con diagnóstico clínico de loxoscelismo cutáneo, demostró que el uso de clorfenamina 30 a $40 \mathrm{mg} /$ día intramuscular o subcutáneos por dos días, seguido de 20 a $30 \mathrm{mg} /$ día durante dos días, seguido por dosis de 10 a $20 \mathrm{mg} /$ día según evolución clínica, se asoció con una reducción del dolor y el edema en los sujetos tratados (reducción del dolor a las 12 horas en 19 sujetos y en todos entre 2 a 10 días; reducción "importante" del edema en 18 sujetos, a las 24 horas de tratamiento).

Efectos adversos. No se describen.

\section{Corriente eléctrica de alto voltaje}

Efectividad. Una serie de $\operatorname{casos}^{85}$ (Nivel IV; calidad grado D; outcome nivel 2), realizado entre 147 sujetos con diagnóstico de loxoscelismo cutáneo confirmado o probable (mediante visualización de la araña o cuadro clínico compatible, respectivamente), demostró que el uso de corriente eléctrica de alto voltaje, aplicada directamente mediante una pieza de mano activada por una batería de $9 \mathrm{Mv}$, en dos pulsos de 40 a $50 \mathrm{Kv}$, separados por 5 a 10 segundos, sería efectiva en detener la progresión local de la mordedura (necrosis o formación de ampollas), y reducir la necesidad de cirugía (ningún sujeto mostró progresión de la lesión cutánea, ni requirió cirugía).

Efectos adversos. No se describen.

\section{Oxígeno hiperbárico}

Efectividad. Una serie de $\operatorname{casos}^{86}$ (Nivel IV; calidad grado D; outcome nivel 2), realizado entre 14 sujetos con diagnóstico de loxoscelismo cutáneo, confirmado mediante cuadro clínico compatible y seguimiento de 1 a 3 días antes del inicio de la intervención, demostró que el uso de oxígeno hiperbárico 2 a 2,5 atmósferas durante 90 minutos, una o dos veces al día, durante un promedio de 7 días, sería efectivo en reducir la tasa de cicatrización anómala y necesidad de injerto (ningún sujeto presentó cicatriz anómala ni requirió injerto). El tratamiento fue aplicado luego de un promedio de 2,6 días.

Efectos adversos. No se describen.

\section{Cirugía}

Efectividad. Un ensayo clínico (Nivel III-1; calidad grado D; outcome nivel 2) ${ }^{91}$, realizado entre 31 sujetos con diagnóstico clínico de loxoscelismo cutáneo (mediante visualización de la araña o cuadro clínico compatible), demostró que el tratamiento quirúrgico precoz (cierre primario o por segunda intención, con o sin injerto), fue inferior al uso de dapsona (dosis y esquema de administración no descrito), en evitar retraso de la cicatrización, o cicatrización anómala (ver más arriba).

Una serie de $\operatorname{casos}^{87}$ (Nivel IV; calidad grado D; outcome nivel 2), realizada entre 33 sujetos con diagnóstico clínico de loxoscelismo cutáneo, demostró que el tratamiento quirúrgico precoz (escisión de lesión cutánea y cierre en segunda etapa), sería, en opinión de los autores, más efectivo que cirugía tardía (escisión y cierre en una o dos etapas), y que el tratamiento no quirúrgico (uso de antimicrobianos, ACTH, antihistamínicos). No se describe el outcome evaluado.

Efectos adversos. No se describen.

\section{Suero anti-loxosceles}

Efectividad. Un ensayo clínico randomizado (Nivel II; calidad grado D; outcome nivel 2$)^{89}$, realizado entre sujetos con diagnóstico de loxoscelismo cutáneo, confirmado por visualización directa de la araña por un entomólogo o investigador experto (sujetos confirmados y analizados 17; sujetos excluidos 78), demostró que no habría diferencias entre el uso de dapsona en dosis de 100 $\mathrm{mg}$ /día, la combinación de suero anti-L. reclusa obtenido de conejos en dosis de 0,2 $\mathrm{ml}$ intralesional y dapsona 100 $\mathrm{mg} /$ día, o sólo suero anti- $L$. reclusa $0,2 \mathrm{ml}$ intra-lesional, en cuanto acortar el tiempo hasta la curación de la herida cutánea (ver más arriba).

Efectos adversos. No se describen.

\section{Corticosteroides}

Efectividad. Una serie de $\operatorname{casos}^{88}$ (Nivel IV; calidad grado D; outcome nivel 2), realizado entre 262 sujetos con diagnóstico clínico de loxoscelismo cutáneo, tratados con múltiples intervenciones, demostró mediante análisis multivariado, que el uso de corticosteroides sistémicos se asociaría a un retraso en la curación de la herida cutánea (ver más arriba).

Efectos adversos. No se describen.

\section{Discusión}

Dapsona. De acuerdo con la evidencia presentada, el uso de dapsona parece ser más efectiva que la utilización de clorfenamina o tratamiento quirúrgico precoz, en el tratamiento de sujetos afectados por loxoscelismo cutáneo. Por otro lado, el uso de dapsona combinado con suero anti- L. reclusa, no parece ser más efectivo que cada tratamiento por separado.

Si bien ningún estudio reportó efectos adversos serios, el uso de dapsona se ha asociado a varios efectos adversos significativos, incluyendo ictericia colostásica, hepatitis, leucopenia, meta-hemoglobinemia y neuropatía periférica 93,94. Asimismo, si bien todos los pacientes tratados con 
dapsona experimentan cierto grado de anemia ${ }^{94}$, esto es especialmente mayor en sujetos con déficit de enzima 6-glucosa fosfato deshidrogenasa. Esta situación debe ser considerada, tanto para los controles de aquellos sujetos tratados con este medicamento, como ante la situación de sujetos tratados empíricamente con este fármaco por diagnóstico de loxoscelismo, en los cuales la presencia de anemia hemolítica puede ser difícil de diferenciar como efecto del medicamento versus el potencial compromiso visceral de la mordedura.

Dado los potenciales efectos adversos de este medicamento, y los efectos sistémicos del veneno en los casos con compromiso visceral, en nuestra opinión este fármaco estaría contraindicado en este grupo de pacientes.

La deficiente calidad de los estudios analizados, determinado fundamentalmente por un proceso de randomización poco detallado, así como un mal manejo de pérdidas y abandonos, y la ausencia de ciego, hace que los resultados derivados de estos estudios sean cuestionables, en cuanto al posible sesgo que pudiese haber sido introducido en la ejecución de los estudios. Por otro lado, la heterogeneidad en la definición de loxoscelismo, así como los variables tiempos post-mordedura en los cuales las intervenciones fueron aplicadas, junto con el delicado balance riesgobeneficio al considerar los potenciales efectos adversos del medicamento, hace que la aplicabilidad de estos hallazgos a la práctica clínica sea limitada.

Clorfenamina. De acuerdo a la información presentada, parece razonable plantear la inferioridad de clorfenamina frente al uso de dapsona, en sujetos con loxoscelismo cutáneo. Sin embargo, nuevamente tanto la limitada validez interna de los estudios, así como la cuestionable aplicabilidad de los resultados, hacen difícil extrapolar una recomendación clínica basado en estos resultados.

Corriente eléctrica de alto voltaje y oxigeno hiperbárico. Ambas alternativas podrían ser efectivas y requieren de más investigación para elaborar conclusiones más concretas. Las series de casos que apoyan su efectividad debiesen ser seguidas de estudios prospectivos controlados a futuro.

Cirugía. De acuerdo a la evidencia presentada, la cirugía precoz, al ser comparada con el uso de dapsona, parece ser inefectiva e incluso perjudicial en este grupo de sujetos. De esta forma, parece razonable recomendar el tratamiento quirúrgico tardío, sólo semanas después de la mordedura, siempre bajo estricta observación y ante un cuadro clínico que amerite la intervención.

Corticosteroides y suero anti-loxosceles. Llama la atención la escasez de información existente respecto a estas dos alternativas terapéuticas, considerando que son de las terapias más recomendadas para el manejo de este tipo de pacientes. Basados en esta revisión, el uso de un suero de origen animal anti-L. reclusa no fue más efectivo que dapsona o la combinación de ambos. Según una serie de casos, el uso de corticosteroides sistémicos pudiese incluso ser perjudicial, retardando el proceso de curación de la herida cutánea.

\section{Conclusiones}

\section{Implicaciones para la práctica clínica}

Varias limitantes surgen en el panorama actual de la investigación clínica en este tema, que hacen difícil o imposible la elaboración de recomendaciones clínicas concretas. Por un lado, existe poca información disponible, siendo la gran mayoría de ésta de calidad deficiente, tanto en cuanto al diseño metodológico empleado, como en la aplicabilidad que estos datos pueden tener en la práctica clínica diaria.

Sin perder de vista lo anterior, parece razonable por el momento, aun pese a la falta de evidencia científica que lo respalde, mantener la recomendación sobre el uso de hielo, inmovilización del área afectada y acudir a un servicio de urgencia ante la sospecha de mordedura por araña genero Loxosceles, idealmente con la araña sospechosa para lograr identificación.

Si bien ninguna de las intervenciones tiene evidencia sólida que respalde su efectividad, según nuestro criterio, el uso de dapsona parece razonable, especialmente en casos de consulta precoz con diagnóstico confirmado (idealmente mediante identificación de la araña). Sin embargo, como dijimos antes, este fármaco no estaría indicado en los casos con compromiso visceral. Del mismo modo, el uso de clorfenamina, aunque según lo descrito, menos efectivo que dapsona, también nos parece una alternativa razonable, aún más considerando el balance riesgo-beneficio entre ambos medicamentos.

Por otro lado, parece prudente mantener el tratamiento quirúrgico como segunda línea, mientras que el uso de oxigeno hiperbárico nos parece una alternativa, según sea la disponibilidad del recurso, sobretodo en aquellos casos de consulta más tardía, dado la mayor ventana terapéutica que parece tener este tratamiento.

Finalmente, no nos es posible establecer recomendaciones respecto uso de corticosteroides, electricidad, ni suero anti-loxosceles. Así, tampoco existe evidencia que permita elaborar recomendaciones para el tratamiento de pacientes con loxoscelismo con compromiso visceral.

\section{Implicaciones para la investigación futura}

No existen estudios clínicos de buena calidad que orienten la toma de decisiones en el manejo de pacientes con loxoscelismo cutáneo y cutáneo-visceral.

Esta ausencia de estudios es llamativa, considerando el 
problema de salud pública que representa esta condición, especialmente en países sudamericanos. Es también llamativa la falta de información respecto a terapias comúnmente utilizadas en este grupo de pacientes, como los corticosteroides o el suero anti-loxosceles, así como la falta de información respecto al manejo de los casos con compromiso visceral.

La ejecución de futuros estudios debiese estar focalizada a encontrar los mejores esquemas terapéuticos, ya sea como monoterapia o combinaciones de las terapias que hasta ahora han demostrado cierta efectividad, como es el caso de dapsona, clorfenamina, suero hiperbárico o aplicación de corriente eléctrica. Por otro lado, es urgente contar con estudios que evalúen la efectividad y seguridad del uso de corticosteroides, fundamentalmente por el alto grado de utilización en la práctica diaria, así como de suero anti-loxosceles, dado su actual utilización pese a la falta de cualquier tipo de evidencia en humanos que apoye su uso, además de la constante presión de la comunidad y medios de comunicación por contar con este tipo de sueros en los servicios de urgencia del país.

Es necesario que la investigación clínica futura se realice en base a ensayos clínicos con adecuada randomización, idealmente ciegos y con adecuado manejo de pérdidas y abandonos, de modo de incrementar la validez interna de éstos. Asimismo, estos estudios debiesen contar con un tamaño de muestra adecuado, de modo de determinar si la ausencia de efectividad encontrada con algunas intervenciones (p. ej dapsona versus suero anti-loxosceles versus tratamiento combinado) se debe a una real ausencia de efecto, o bien a baja potencia de los estudios realizados hasta ahora.

Es menester que la futura investigación considere criterios de inclusión y definición de caso explícitos y reproducibles, así como también es necesario que estos estudios definan ventanas de tratamiento explícitas, de modo de establecer hasta qué momento de evolución de la mordedura son útiles y seguras, las distintas estrategias terapéuticas.

Agradecimientos. A la Dirección de Investigación de la Escuela de Medicina de la Pontificia Universidad Católica, por el financiamiento entregado a esta investigación, mediante la adjudicación de un Proyecto de Investigación para Residentes Becarios de la Escuela de Medicina.

\section{Resumen}

Introducción: El loxoscelismo constituye un importante problema de salud pública, en el que no existen intervenciones terapéuticas estándares. Objetivo: Encontrar y analizar la evidencia científica relacionada al manejo de esta patología. Método: Revisión sistemática de estudios clínicos. La búsqueda incluyó múltiples bases de datos, búsqueda manual de referencias, y contacto con expertos. Resultados: Tres ensayos clínicos de pobre calidad metodológica fueron identificados. Uno de ellos (n =31), encontró que el uso de dapsona se asoció con menos complicaciones locales, que el tratamiento quirúrgico. Un segundo estudio $(n=46)$, encontró que el uso de dapsona fue superior a clorfenamina, en mejorar la evolución de las lesiones cutáneas. Un tercer estudio $(n=95)$, no demostró diferencias entre el uso de dapsona oral, suero anti-Loxosceles reclusa o una combinación de ambos. Discusión. No existe suficiente evidencia de buena calidad, que permita orientar la toma de decisiones, en el manejo de sujetos con loxoscelismo cutáneo o cutáneo-visceral.

\section{Referencias}

1.- Ríos J, Pérez M, Sánchez M, Bettini M, Mieres J, Paris E. Caracterización clínicoepidemiológica telefónica de la mordedura por araña de rincón, en un centro de información toxicológica de Chile, durante el año 2005. Rev Méd Chile 2007; 135: 1160-5.

2.- Swanson D L, Vetter R S. Loxoscelism. Clin Dermatol 2006; 24: 213-21.

3.- Gertsch W J, Ennik F. The spider genus Loxosceles in North America, Central America and the West Indies (Araneae loxoscelidae). Bull Am Mus Nat Hist 1983; 175: 264-360.

4.- Van Den Berg C W, De Andrade R M, Magnoli F C, Marchbank K J, Tambourgi D V. Loxosceles spider venom induces metalloproteinase mediated cleavage of $\mathrm{MCP} / \mathrm{CD} 46$ and $\mathrm{MHCI}$ and induces protection against C-mediated lysis. Immunology 2002; 107 : 102- 10.
5.- Futrell J M. Loxoscelism. Am J Med Sci 1992; 304: 261-7.

6.- Southcott R V. Spiders of the genus Loxosceles in Australia. Med J Aust 1976; 1: 406-8.

7.- $\quad$ Saraco S, De Roodt A. Envenenamiento por animales ponzoñosos. Loxoscelismo. ATA 2005; 67: 45-9.

8.- Da Silva P, Bertoni R, Appel M, Mangili O, Gremsky W, Sanches S. Brown spider and loxoscelism. Toxicon 2004; 44: 693-709.

9.- Young A R, Pincus S J. Comparison of enzymatic activity from three species of necrotizing arachnids in Australia: Loxosceles rufescens, Badumna insignis and Lampona cylindrata. Toxicon 2000; 39: 391-400.

10.- Foradori M J, Smith S C, Smith E, Wells R E. Survey for potentially necrotizing spider venoms, with special emphasis on Cheiracanthium mildei. Comp Biochem Physiol C Toxicol Pharmacol 2005; 141: 32-9.

11.- Bodner M R, Callahan M, Binford GJ. Evolu- tionary origin and loss of sphingomyelinase D in the Sicarius and Loxosceles lineages. 29th Annual Meeting of Amer Arachnol Soc. Akron OH, 29 Jun 2005.

12.- Schenone H. Cuadros tóxicos producidos por mordeduras de araña en Chile: latrodectismo y loxoscelismo. Rev Méd Chile 2003; 131: 437 44.

13.- Tambourgi D, Paixao-Cavalcante D, Goncalves R, Fernándes-Pedrosa M, F, Morgan P, Van De Berg C. Loxosceles sphingomyelinase induces complement- dependent dermonecrosis, neutrophil infiltration, and endogenous gelatinase expression. J Invest Dermatol 2005; 124: 725 31.

14.- Schenone H. Cutaneous loxoscelism with edematous predominance. Bol Chil Parasitol 1998; 53 (3-4): 78-83.

15.- Hogan C, Barbaro K, Winkel K. Loxoscelism: Old obstacles, new directions. Ann Emerg Med 2004; 44: 608-24. 
16.- Swanson D, Vetter D. Bites of brown recluse spiders and suspected necrotic arachnidism, $\mathrm{N}$ Engl J Med 2005; 325: 700-7.

17.- Manríquez J J, Silva S. Dermatología basada en la evidencia: importancia y conceptos generales. Piel 2006; 21 (3): 118-23.

18.- Manríquez J J, Silva S. Dermatología basada en evidencia: revisiones sistemáticas y meta-análisis. Piel 200621 (7): 323-8.

19.- Manríquez JJ, Silva S. Dermatología basada en la evidencia: ¿un enfoque posible? Revista Medicina Cutánea Iberoamericana 2006; 34 (5): 187-97.

20.- Letelier L M, Manríquez J J, Rada G. Systematic reviews and meta-analysis: are the best evidence? Rev Med Chile 2005; 133 (2): 246-9.

21.- Manríquez J J. A highly sensitive search strategy for clinical trials in Literatura Latino Americana e do Caribe em Ciências da Saúde (LILACS) was developed. J Clin Epidemiol 2008; 61(4): 407-11.

22.- Manríquez J J, Villouta F, Williams H. Evidence based dermatology: Number needed to treat and it relation to others risk measures. J Am Acad Dermatol 2007; 56 (4): 664-71.

23.- National Health and Medical Research Council. Disponible en http://www.nhmrc.gov.au (accedido 1/12/2007).

24.- Cochrane Reviewer Handbook. Disponible en http://www.cochrane.org/resources/handbook/. (accedido el 1/12/2007)

25.- Von Elm E, Altman D G, Egger M, Pocock S J, Gotzsche P C, Vandenbroucke J P; for the STROBE Initiative. The Strengthening the Reporting of Observational Studies in Epidemiology (STROBE) statement: guidelines for reporting observational studies. J Clin Epidemiol. 2008; 61 (4): 344-9.

26.- En: Insuficiencia renal aguda por Loxoscelismo: estudio de 14 casos presentados en el Hospital Cayetano Heredia (Kemper Vásquez RS, ed). s.1: UPCH. Facultad de Medicina Alberto Hurtado, 1987.

27.- En: Loxoscelismo en niños: estudio retrospectivo de pacientes hospitalizados entre $1970 \mathrm{y}$ 1990 en los Servicios de Pediatría del Hospital Cayetano Heredia e Instituto Nacional de Salud del Niño (Mangiante Russo RMI, ed). s.l: UPCH. Facultad de Medicina Alberto Hurtado, 1991.

28.- En: Loxoscelismo en adultos morbi - mortalidad $\mathrm{y}$ factores asociados al desarrollo de la forma viscero - hemolítica (Quispe Avendaño AJ, ed). Arequipa: s.n, 1997.

29.- En: Loxoscelismo no estado do Paraná: análise epidemiológica dos acidentes causados por Loxosceles Heinecken \& Lowe, 1832, no período de 1993 a 2000 (Silva EMd, ed). Rio de Janeiro: s.n, 2002.

30.- Anderson P C. Loxoscelism threatening pregnancy: Five cases. Am J Obstet Gynecol 1991;
165: 1454-6.

31.- Arias Obarrio H J. Loxoscelismo cutáneonecrótico simple (a propósito de cinco observaciones). Arch Argent Dermatol 1982; 32 : 223-32.

32.- Arnold R E. Brown recluse spider bites: five cases with a review of the literature. JACEP 1976; 5: 262-4.

33.- Barretto OCdO, Cardoso J L, Cillo Dd. Viscerocutaneous form of loxoscelism and erythrocyte glucose-6-phosphate deficiency. Rev Inst Med Trop Säo Paulo 1985; 27: 264-7.

34.- Barrio A, Ibarra-Grasso A. Cases of severe loxoscelism which took place in Buenos Aires and its surroundings in the last years. Mem Inst Butantan 1966; 33: 809-19.

35.- Benavides M I, Moncada X. The treatment of cutaneous loxoscelism with dapsone. Rev Med Chil 1990; 118: 1247-50.

36.- Bravo M. Study of anti-loxosceles serum action on hemolytic and ulcero-necrotic cutaneous effects of Loxosceles laeta venom. Rev Med Chil 1994; 122: 625-9.

37.- Braz A. Development and evaluation of the neutralizing capacity of horse antivenom against the Brazilian spider Loxosceles intermedia. Toxicon 1999; 37: 1323-8.

38.- Burton K G. Nitroglycerine patches for brown recluse spider bites. Am Fam Physician 1995; 51: 1401.

39.- Cacy J, Mold J W. The clinical characteristics of brown recluse spider bites treated by family physicians: an OKPRN Study. Oklahoma Physicians Research Network. J Fam Pract 1999; 48 : 536-42.

40.- Cardoso J L. Loxoscelismo: estudo de 242 casos (1980-1984). Rev Soc Bras Toxicol 1988; 1: 58-60.

41.- Cermeño J, Carpio N, Salazar N. Aracnoidismo en el Hospital Universitario Ruíz Páez estado Bolívar Venezuela: y revisión de la literatura. Rev Soc Venez Microbiol 2004; 24: 95-7.

42.- Clowers T D. Wound assessment of the Loxosceles reclusa spider bite. J Emerg Nurs 1996; 22: 283-7.

43.- Cohen N. Dermonecrotic loxoscelism in the Mediterranean region. J Toxicol-Cutaneous Ocular Toxicol 1999; 18: 75-83.

44.- Dasilva M V. Utilization of exchange-transfusion procedure in serious human loxoscelism. Rev Institut Med Tropical S Paulo 1988; 30: 259-63.

45.- Dyachenko P, Ziv M, Rozenman D. Epidemiological and clinical manifestations of patients hospitalized with brown recluse spider bite. J Eur Acad Dermatol Venereol 2006; 20: 1121-5.

46.- Elbahlawan L M, Stidham G L, Bugnitz M C, Storgion S A. Quasney MW. Severe systemic reaction to Loxosceles reclusa spider bites in a pediatric population. Pediatr Emerg Care 2005; 21 (3): 177-80.

47.- Ersek R A. Treatment of spider bites with silver- impregnated porcine xenografts. Tex Med 1985; 81: $32-5$

48.- F O S Barbaro K C, Fan H W. A clinical and epidemiological study of Loxosceles spider envenoming in Santa Catarina, Brazil. Trans R Soc Trop Med Hyg 1998; 92 (5): 546-8.

49.- Farace F, Lissia M, Mele A, Masia D R, Rubino C. Local cutaneous arachnidism: A report of three cases and their management. J Plastic, Reconstructive Aesthetic Surg 2006; 59 (2): 197-201.

50.- Felix R. Mordedura de araña loxosceles: en pacientes pediátricos. Rev Serv Sanid Fuerzas Polic 1987; 48: 43-6.

51.- Fishman T D. Wound assessment and evaluation. Brown recluse spider bite. Dermatol Nurs 1999; 11: 290-1.

52.- González C. Acute renal failure in viscerocutaneous loxoscelism: 11 cases. Rev Med Chil 1986; 114: 1155-9.

53.- Gutiérrez Puelma J, Sagua Franco H. Loxoscelism in children. Analysis of 15 cases. Rev Chil Pediatr 1979; 50: 21-7.

54.- Hailey D. Hyperbaric oxygen therapy - recent findings on evidence for its effectiveness. Update (Structured abstract). 2003.

55.- Iribarren O. Loxoscelism in the province of Valdivia (X Region, Chile). Clinical study of 9 cases. Bol Chil Parasitol 1987; 42: 68-71.

56.- King L E Jr, Rees R S. Dapsone treatment of a brown recluse bite. JAMA 1983; 250: 648.

57.- Leach J, Bassichis B, Itani K. Brown recluse spider bites to the head: Three cases and a review. Ear, Nose and Throat J 2004; 83(7): 465-70.

58.- Lee M S, Bernstein H H. Immunizations, neonatal jaundice, and animal-induced injuries. Curr Opin Pediatr 2005: 418-29.

59.- Malaque C M. Clinical and epidemiological features of definitive and presumed loxoscelism in Sao Paulo, Brazil. Rev Inst Med Trop Sao Paulo 2002; 44: 139-43.

60.- Marques-da-Silva E. Loxosceles spider bites in the state of Paraná, Brazil: 1993-2000. J Venom Anim Toxins Incl Trop Dis 2006; 12: 110-23.

61.- Martino O A. Emponzonamiento humano provocado por venenos de origen animal. Estudio epidemiologico clinico y experimental. Arch Argent Dermatol 1981; 31: 41-7.

62.- Maya Rodríguez L E. Hemodialysis with artificial kidney in the treatment of acute renal insufficiency from loxoscelism. An Fac Med Lima 1964; 47: 419-39.

63.- McClure D N. Necrotising arachnidism treated with hyperbaric oxygen. Med J Aust 1995; 163: 51-2.

64.- Medical Services Advisory C. Hyperbaric oxygen therapy (Structured abstract), 2001.

65.- Osborn C D. Treatment of venomous bite by high voltage direct current. J Okla State Med Assoc 1990; 83: 9-14.

66.- Ramires E N. Evaluation of the efficacy of 
vacuum cleaners for the integrated control of brown spider Loxosceles intermedia. J Venom Anim Toxins Incl Trop Dis 2007; 13: 607-19.

67.- Sams H H. Nineteen documented cases of Loxosceles reclusa envenomation. J Am Acad Dermatol 2001; 44: 603-8.

68.- Schenone H. Loxoscelismo en pediatría: Región Metropolitana Chile. Rev Chil Pediatr 2001; 72: 100-9.

69.- Schenone H. Latrodectism and loxoscelism incidence in Chile, clinical characteristics, diagnosis, treatment and prevention. Mem Inst Butantan 1966; 33: 207-11.

70.- Schenone H. Cutaneous loxoscelism with edematous predominance. Bol Chil Parasitol 1998; 53: 78-83.

71.- Schenone H, Prats F. Arachnidism by Loxosceles laeta. Report of 40 cases of necrotic arachnidism. Arch Dermatol 1961; 83: 139-42.

72.- Schenone H. Epidemiology and clinical course of loxoscelism. A study of 133 cases caused by the bite of the corner spider (Loxosceles laeta). Bol Chil Parasitol 1975; 30: 6-17.

73.- Schenone H. Loxoscelism in Chile - epidemiological, clinical and experimental studies. Rev Instit Med Trop Sao Paulo 1989; 31: 403-15.

74.- Skinner M W, Butler C S. Necrotising arachnidism treated with hyperbaric oxygen. Med J Austr 1995; 162: 372-3.

75.- Soto L. Tres casos pediátricos de loxoscelismo cutáneo en zona rural de Valdivia: Chile. Bol
Chil Parasitol 1991; 46: 74-6.

76.- Valverde López J. Aspectos clínicos y epidemiológicos del loxoscelismo, Hospital Regional Docente de Trujillo, enero 2001 a noviembre 2003. Folia Dermatol Perú 2003; 14: 15-9.

77.- Villanueva E. Hyperbaric oxygen therapy (Structured abstract). 2000.

78.- Wright S W. Clinical presentation and outcome of brown recluse spider bite. Ann Emerg Med 1997; 30: 28-32.

79.- Zavaleta Alfaro R L, Alvarado Ganoza G. Loxoscelismo en niños: algunos aspectos clínicos y epidemiológicos en el Hospital Belén de Trujillo. Diagnóstico (Perú) 1987; 20: 84-6.

80.- Svendsen F J. Treatment of clinically diagnosed brown recluse spider bites with hyperbaric oxygen: a clinical observation. J Ark Med Soc 1986. 83: 199-204.

81.- Maguiña Vargas C P. Uso de azúcar granulada en úlcera cutánea moderada a severa por loxoscelismo. Folia Dermatol Perú 2004; 15: 87-93.

82.- Escalante Galindo P. Loxoscelismo local dermonecrótico en niños mordidos por la araña Loxosceles reclusa (araña "violinista"). Gac Méd Méx 1999; 135: 423-6.

83.- Maguiña Vargas C. La dapsona (DDS) en el loxoscelismo cutáneo. Diagnóstico (Perú) 1987; 20: 58-64.

84.- Schenone H. Estudio de 27 casos de loxoscelismo. Bol Chil Parasitol 1959; 14: 7-13.

85.- Osborn C D. Treatment of spider bites by high voltage direct current. J Okla State Med Assoc 1991; 84: 257-60

86.- Maynor M L, Abt J L, Osborne P D. Brown recluse spider bites: beneficial effects of hyperbaric oxygen, J Hyperb Med 1992; 7: 89-102.

87.- Auer A I, Hershey F B. Proceedings: Surgery for necrotic bites of the brown spider. Arch Surg 1974; 108: 612-8

88.- Mold J W, Thompson D M. Management of brown recluse spider bites in primary care. J Am Board Fam Pract 2004; 17: 347-52.

89.- Rees R. The diagnosis and treatment of brown recluse spider bites. Ann Emerg Med 1987: 945 9.

90.- Maguiña Vargas C, Gotuzzo Herencia E, Álvarez H. Nuevos esquemas terapéuticos en loxoscelismo cutáneo en Lima, Perú. Folia Dermatol Perú 1997; 8: 23-30.

91.- Rees R S. Brown recluse spider bites. A comparison of early surgical excision versus dapsone and delayed surgical excision. Ann Surg 1985: 659-63.

92.- Schulz K F, Chalmers I, Hayes R J. Empirical evidence of bias. J Am Med Acad 1995; 273 : 408-12.

93.- Iserson K V. Methemoglobinemia from dapsone therapy for a suspected brown spider bite. J Emerg Med 1985; 3: 285-8.

94.- Pfeiffer C, Wozel G. Dapsone and sulfones in dermatology: overview and update. J Am Acad Dermatol 2003; 48: 308-9. 